\title{
The Dynamics of Cognitive Performance: What Has Been Learnt from Empirical Research in Science Education
}

\author{
DIMITRIOS STAMOVLASIS \\ Aristotle University of Thessaloniki (Greece)
}

This paper discusses investigations in science education addressing the nonlinear dynamical hypothesis. Learning science is a suitable field for applying interdisciplinary research and predominately for testing psychological theories. It was demonstrated that in this area the paradigm of complexity and nonlinear dynamics have offered theoretical advances and better interpretations of empirical data. Research showed that besides linear modes of behavior, sudden transitions occur in cognitive performance and this has questioned basic theoretical and epistemological assumptions. The neo-Piagetian framework and motivational theories offering constructs for serving as predictors in various model are the local theories which are embraced by the CDS meta-theory. Sudden transitions are modeled by catastrophe theory (CT) the analyses of which reveal the crucial role of certain variables, namely the bifurcation factors. Beyond a critical value of the bifurcation factor, the state variable splits into two-attractor regions and becomes bimodal. The bifurcation effect induces uncertainty and unpredictability in the system, which oscillates between two states entering the regime of chaos. Then in state variables such as learning outcomes and achievement, sudden transitions from success to failure are expected. Catastrophe theory explains unexpected phenomena associated with school failure, dropouts, illicit behaviors, sudden attitude change, and creativity. Moreover CT could contribute in elucidating theoretical debates and conflicting empirical evidences.

Educational research aims to explore the factors affecting school performance and students' academic and social behavior. School improvement is highly determined by the validity of the educational theories. These theories are being constantly built through empirical research and thus methodology is crucial. Research methods have to be appropriate for probing systems' properties and this is not always met by linear approaches when exploring systems that are not mechanical and stable in time. The new paradigm proposing the theory of Complex Dynamical Systems (CDS) as a meta-theoretical framework to describe and explain human behavior has already been established in other disciplines (e.g. Guastello, Koopmans \& Pincus, 2009). In educational research, analogous advances within the new paradigm has also been presented in 
collective outlets and signified a new era in thinking, designing and realizing empirical research (Stamovlasis \& Koopmans, \& 2014; Koopmans \& Stamovlasis, 2016). These endeavors during the last decade have accumulated a large body of knowledge comprising of the latest methodological advances, the theoretical development and improved educational practices that can inform teachers, researcher and policy makers.

In science education, interdisciplinary research, especially with contributions from psychology has always been fruitful, because it entails basic research and this is what improves theory.

What new the CDS brought to theories in science education? Research, so far, has revealed the factors affecting students' achievement, and we know most of correlations, between performance and individual differences or performance and environmental constraints. However, these relationships are expressed in linear terms, that is, small variation in our predictors will lead to small change in the dependent variable and large input will lead to large outcomes. This is what an unsuspecting researcher believes, if he or she is attached to traditional paradigm. Nevertheless, behaviors in human systems do not always follow patterns that can be described with linear equations. These behavioral patterns are expected to be complex, that is, they cannot be straightly reduced to their components and since are dynamical, are also nonlinear. The notion of dynamics is associated with the time and thus studying behavior over time is what the ontology of these systems demands from an appropriate research methodology. The CDS framework provides various concepts and tools to describe complex systems changing with time. Such concepts are attractors, fractals, chaos, catastrophes, bifurcations, or catastrophes to mention a few (Prigogine \& Stengers, 1984). Descriptions with the use of the above terms have been realized with metaphorical language in the past and without observations, measurements and data analysis via the proper methodology capturing the phenomena under investigation. Thus, if education is considered as an empirical science - not as social philosophy -- research in this field has to be carried out by means of methodological assets of empirical sciences. Following the new paradigm, we consider that straight lines are the exceptions and nonlinearities are more likely to occur. The tools of CDS are capable to capture them, and the nonlinear hypothesis in educational research expresses precisely the aim to investigate them. The scope is to explain them and provide a better understanding of reality. Testing nonlinear hypotheses follows Popper's epistemology, emphasizing the falsifiability of theoretical accounts and their rejection in the light of empirical evidences. The manifestation of nonlinearity is not always evident; however when it appears, it has important implications for both theory and practice. A brief illustration of what has been learnt from empirical research in science education is provided in the following sections. The added values of CDS perspective can be appreciated if the limitations of traditional approaches and what is sought in the new paradigm are understood.

\section{On the Limitations of Linear Empirical Methods}

There is no doubt that the traditional methodologies based on linear statistics have contributed a great deal in developing theories and in understanding the phenomena associated with learning and reaching. Even though, the advances of statistical tools permits testing a wide range of hypotheses, there are still core epistemological and methodological issues that make inference difficult. In order examine those issues more closely, some comments on the experimental settings and the cross-sectional studies will be made next. 
Experiments are considered as the only suitable approach to establish causality. This is achieved by controlling a number of variables and letting some others vary, so that the outcome can be surely attributed to the factors under examination. The randomized control trials (RCT) and related designs such as quasi-experimental ones in educational settings and any social process are questionable, at least insofar as the nonlinear hypothesis is concerned. The main weakness of RCT in educational experiments is that the process under investigation is, in fact, ignored and the system is faced as a 'black box' Koopmans (2014a; 2014b). However, cognitive and social phenomena taking place in educational settings are not mechanistic processes where the effect of each contributed component can be linearly expressed in the final result. In addition, these processes are not deterministic ones, and the dynamical character prevails as the main ontological feature, which has been ignored. Thus, the main question is how, one may establish a process explanation of causality.

Next, consider cross-sectional studies based on linear statistics, which analyze measured variables e.g. students' achievement, assuming that certain presuppositions, such as the central limit theory or normal distribution, hold. These, however, are not always true. Often, deviation from normality is observed, while in multivariate models the errors are not independent (iid-errors), as they are assumed. A fundamental limitation of linear modelling is the low predictability, and this is not due merely to statistical presuppositions which might not hold or to the variables ignored by the theory, but it is deeply epistemological (Stamovlasis, 2010). In simple language, it is impossible to infer about a flow by taking a 'snap shot'.

Moreover, another question, that the traditional linear epistemology fails to address, is about the origin and prediction of sudden changes, often observed in behavior, which are rather unanticipated within the local explanan framework. Phenomena, such as students' failure, dropouts, and changes in feelings, attitude, or behavior are frequently observed in school processes. They are what in the mathematical language called discontinuities and along with smooth and linear changes co-occur at all levels of complexity in educational systems. What is needed is the research methodology to capture them, and on the other hand the theoretical interpretation.

\section{Modeling Abrupt and Unexpected Behaviors}

Complex Dynamical Systems framework provides an extremely useful modeling approach for nonlinear changes via catastrophe theory (CT). The application of CT is valuable because it is easy and allows testing nonlinear changes using cross-sectional data. The latter, makes CT popular because researchers can re-examine and re-analyze data collected in the past and reconsider the linear modeling in the light of potentially new results, if any. Moreover, given the new findings, local theories can be enriched and interpretation can be improved.

CT describes discontinuous changes between stable states in a system observed under gradual increases in a number of independent variables named the control variables. The most popular is the cusp model (Guastello, 2001; 2002) which describes the changes between two qualitatively different states by two controlling variables, the asymmetry, a and the bifurcation, $\mathrm{b}$, factors respectively. The cusp model is described mathematically by a potential function:

$$
U(y, a, b)=\frac{1}{4} y^{4}-\frac{1}{2} b y^{2}-a y
$$

Where, $y$ is the dependent variable. When bifurcation variable, $\mathbf{b}$, has low values, then the 
system is rather stable and a linear relationship best describes the link between the asymmetry variable and the dependent variable, that is, behavior changes gradually and smoothly as a function of a. However, when the bifurcation variable takes on high values the behavior becomes bimodal. The model is three dimensional, but it can be perceived in two dimensions, if one considers the change in behavior as a function of $\mathbf{b}$, which actually captures the nonlinear phenomenon. A schematic representation of a bifurcation is depicted in Figure 1. Beyond a threshold the system is no longer linear. Two states or behavioral modes appear in the dependent variable, expressed by the bimodality in the corresponding probability density function. In the CDS language these states are the attractors of the system. Behavior is permitted to vary only within one of the two attractors, while changing attractor entails a jump from one attractor to the other. The observed discontinuous behavior introduces nonlinearity, turbulence and unpredictability in the system, pushing the behavior towards the chaotic regime.

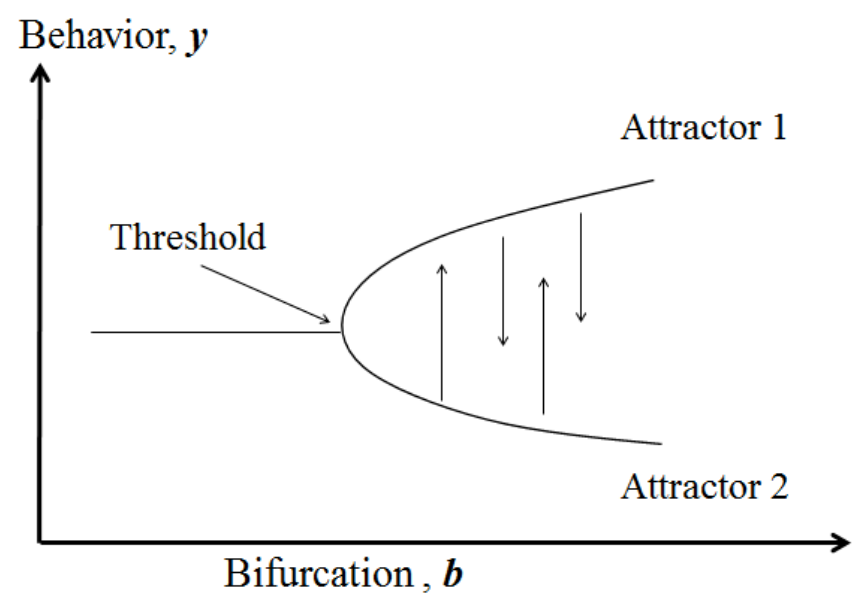

Figure 1. A schematic representation of a bifurcation: Beyond a threshold value of the bifurcation the system possesses two states or behavioral modes (attractors) and the permitted behavior is only the transition between the two attractors.

From a statistical point of view there is a number of modeling approaches, which are based either on the probability density function (pdf) of equation 1 or on its derivative. A usual method with cross-sectional data is based on the probability density function, while as optimization method the least squares (Guastello, 2011) or maximum likelihood (Cobb, 1998) can be implemented. The maximum likelihood method performed in $\mathrm{R}$ cusp package (Grasman, van der Maas, \& Wagenmakers, 2009). In this method the statistical evaluation model fit was based on pseudo-R2 statistics and on AIC, AICc and BIC indices (Akaike's criterion, Akaike's criterion corrected for small samples and Bayes's information criterion, respectively). The likelihood ratio chi-square is also utilized to compare the fit of the cusp models and the linear counterparts. The other approach based also on probability density function uses least square procedures and can be performed with an ordinary statistical package (Guastello, 2011). In this method the main criterion of model fit, besides the statistical significance of the coefficients, is the $\mathrm{R} 2$ values, which denotes the percentage of variance explained and it is a useful index to compare the cusp with the alternatives. Since the two procedures use different optimization algorithms and different criteria for model fit, the 
implementation of both it is recommended to provide further support for the empirical evidence and conclusions related to the hypotheses in question.

A lucid presentation of catastrophe theory, the statistical modelling and the epistemological issues concerning educational research related to nonlinearity can be found Stamovlasis (2016). An important issue when applying a catastrophe model is the appropriate choice for the variables implemented as controls. This is a theory-driven decision and should be based on a deeper understanding of the role of the candidate variables. In general, variables having strong linear association with the dependent variable are suitable for asymmetry factors, while for bifurcation factors, appropriate candidates are moderator variables. Asymmetry and bifurcation factors belong to opposite or antagonistic processes or mechanisms. The selected variables could be of any type, cognitive or affective in nature, individual differences or group characteristics, depending on the local theory implemented to interpret the process under investigation.

Catastrophe theory (Thom, 1975) has been applied in psychology and behavioral science. As characteristic works the connection of CT to Piagetian stagewise development (Molenaar \& Oppenheimer, 1985; Van der Maas \& Molenaar, 1992), to motivation and academic performance (Guastello, 1987), attitude change (van der Maas, Molenaar \& van der Pligt, 2003), modeling cognitive overload phenomena (Stamovlasis, 2006, 2011), could be mentioned, while contemporary literature is continuously enriched.

\section{Nonlinear Dynamics and Complexity in Science Education Research}

\section{On Contemporary Science Education}

Science education research focuses on students' leaning difficulties in conceptual understanding of physical and chemical phenomena and problem solving. There are many challenging areas, i.e. teaching the material world in terms of atoms and molecules and problem solving in physics, i.e. how to describe moving objects and predict their positions given their velocity or acceleration. In mathematics likewise, challenging problem solving, e.g., in geometry where task are non-algorithmic is sought for interpretations of students' difficulty.

The large amount of work carried out in order to investigate the teaching and learning difficulties in various content areas has revealed a wide range of common student errors and misconceptions which have been found to persist even after focused teaching interventions. The analysis of the results and their interpretation in any research, demands a theoretical framework. In science and mathematics education there are various schools of thought, which have developed not only a framework of understanding related phenomena, but also specific methodological approaches that are directly associated with data analysis. It is important to stress here that school improvement is highly determined by the efficiency and the validity of the theories applied, which are built through empirical research. Thus, methodology and its epistemology have a crucial role. Moreover, in education all theoretical premises have been traditionally borrowed from other discipline and mainly from psychology. For instance, in learning sciences, one trend is based on conceptual change theories and focused on the nature of students' naïve ideas. Various hypotheses have been posted on the nature of students' mental representations before they acquire the scientific knowledge. On this matter, two antithetic theories prevail: The first states that pupils' supposedly naïve knowledge is stable and coherent, and thus it resembles to a "scientific" theory (e. g. Chi, 2005; Vosniadou \& 
Brewer, 1992). The second one states that the students' naïve knowledge is rather an incoherent structure that consists of multiple quasi-independent elements known as phenomenological primitives or p-prims; when learning is attained these elements are organized towards the scientific view (e.g., Clark, 2006; diSessa, 2006; diSessa, Gillespie, \& Esterly, 2004; Harrison, Grayson, \& Treagust, 1999). It is important to note some key epistemological considerations that the two theoretical perspectives have fostered, however, arbitrarily or intuitively from the beginning of their development. The first, the mental model theory of conceptual change has fostered an architecture metaphor for conceptual change. Conceptual change progresses linearly through distinct intermediate steps by replacing flawed element with correct ones, and ultimately reaches the correct scientific knowledge. The second perspective, posits that elements of knowledge, under certain circumstance, during educational process, are organized towards the science view, and do not consider the hypothesized distinct phases, which represent coherent mental state. These hypothetical stable mental models have been suspected for learning difficulties, since they are supposedly resistant to change. Both of the above theories consider that difficulties arise from the subject matter itself, without however, providing explanations for their origin or correlating them with independent variables.

In another school of thought, researcher consider psychological theories, such as information processing models and neo-Piagetian theories, which view cognitive processes as driven by mental resources explaining variation in performance on cognitive tasks. An example is Pascual-Leone's theory of constructive operators (TCO; Pascual-Leone 1969, 1970). According to TCO, cognitive performance is the responsibility of a variety of constructive operators, each of which performs a specific function: The M-operator deals primarily with mental capacity, the $\mathrm{C}$ operator with content knowledge, the L-operator with logical operations such as conservation and formal logic, the F-operator with field dependence/independence, and so on. Research has supported the construct validity of TCO, since these operators correspond to mental resources activated during cognitive tasks.

The main advantage of this framework is that provides the means for operationalizing the theoretical constructs. The most known psychometric variables measured at the behavioral level are the information processing capacity (WM or M-capacity), logical thinking, field dependence/independence, or convergent/divergent thinking. These have been proven to play a significant role in a wide range of tasks, and they affect students' performance in learning science (e.g. Lawson \& Thompson, 1988; Tsaparlis \& Angelopoulos, 2000; Tsaparlis, 2005).

Last, but not least, besides the cognitive constructs, motivational variables, taken from achievement goal theory have been implemented as predictors and empirical finding have supported their role in learning and school processes (Gonida \& Stamovlasis, 2016).

\section{Catastrophe Theory in Science Education Research}

A series of investigations has examined the applicability of catastrophe theory and attempted to build bridges between educational research and nonlinear dynamics. Students' academic achievement was used as the depended measure predicted by neo-Piagetian constructs. The choice of the neo-Piagetian framework was actually made because the theory from its basic foundation implies an optimization process which assumes equilibrium states of the system, and that is compatible with catastrophe theory (Van der Maas \& Molenaar, 1992) Moreover, neo-Piagetian constructs representing mental resources involved in cognitive processes are in fact protagonist factors, and especially in science leaning, where often high order cognitive operators are demanded, these variables were found to explain considerable portion of the 
variance. The research questions that are posted in catastrophe theory analysis are associated with testing nonlinear hypotheses in educational context. Specifically, the working hypothesis behind these designs is to predict students' achievement in understanding and explaining chemical and physical phenomena. In these cognitive processes, which are dynamical processes, nonlinear and discontinuous changes might occur. That is, bifurcations and hysteresis effects in students' achievement are observed, where certain variables act as bifurcation factors in combination with factor linearly correlated with the behavior. Note that the cusp catastrophe model employs two controls, which in fact are latent variables, operating within opposite mechanisms. The latent nature of the controls, according with contemporary psychometric theory, demands a number of observables, which contribute to hypothesized latent attribute responsible for the splitting of the state variable. Thus, in practice, there are many candidates contributing to bifurcation process and their identification could be achieved through statistical analyses.

The application of cusp models demonstrate that both linear and nonlinear changes in behavioral variable might be expected and the pattern of these changes can be described by the two control variables $b$ and $a$. At low values of $b$, changes are smooth and linear. At high values of $b$ and beyond a threshold changes are discontinuous. This is a dynamic effect and indicates sensitivity of the parameters, that is, small differences in a and /or b, may lead to sudden shifts between the two attractors representing modes of behavior. In the school context, considering achievement as the state variable, these behavioral modes represent success and failure, while sudden transitions between them are observed according to the model under examination.

Variables that have been identified in previous research as asymmetry are: previous knowledge, working memory capacity, M-capacity, developmental level, and mastery goal orientation. These of course are operating as such in relation to the nature of the mental task. For instance, in problem solving, information processing capacity is highly associated with performance. The asymmetry variables are already known from traditional studies as linearly correlated with outcomes. This linearity is not preserved in all tasks and in the whole value range of the splitting variable. A threshold exists beyond which smooth changes turn into abrupt phenomena. The existence of variables acting as bifurcation destroys linear relationships if the nature of the task (environment) differs. For example, field dependence/independence can act as bifurcation factor if certain amount of 'noise' exists in the data (Stamovlasis, 2006). In educational research, a general goal would be the identification of spitting variables and the conditions under which the system enters the area of unpredictability.

Variables such as field dependence/independence, logical thinking, convergent or divergent thinking, have been identified as bifurcation factors. Moreover, in school context or antagonistic situations, where motivation is a driving force, goal orientation constructs appear to be determinants of school achievement. Some variables, e.g. performance avoidance goal orientation have been found to induce bifurcation effects in a number of disciplines (Stamovlasis \& Gonida, 2017).

The knowledge resulting from the above research endeavor is two-fold as far as the theory development and its implications for practice are concerned. First the identification of bifurcation variables exemplifies that those variables are disruptive is the sense that beyond a critical value they induce nonlinear effects, uncertainty, and chaos. Teachers should be aware about these phenomena and explain students' difficulties accordingly, while in the classroom 
the adoption of suitable approaches might facilitate the desirable shifts and constructive 'catastrophes' (Stamovlasis, 2006). On the other hand knowing the role of such variables provides at a general theoretical level sophisticated interpretations of unexpected phenomena, such school failures, dropouts or sudden behavioral changes, and improves decision making on rehabilitation actions.

The most significant implication of above is that bifurcations and hysteresis effects are the signatures of chaos and complexity. The nonlinear findings suggest that the system under investigation is driven by self-organization process (Nicolis \& Nicolis, 2007). The outcome of those mental processes is an emerging phenomenon and this has an implication for the definition of learning. Processes, such as those involved in conceptual change, learning, problem solving are creative processes, where the outcome emerges from a nonlinear dynamical process. For science education, this carries an important message showing which processes lead to real conceptual understating, contrary to those which correspond to row or 'parrot learning', and suggests re-consider teaching processes an curricula design (Stamovlasis, 2011; 2016).

Returning to the aforementioned theories of conceptual change, nonlinear dynamics makes a contribution to the ongoing discussion on the nature of children's naïve knowledge. The theory of mental models has been challenged by new methodological approaches, such as Latent Class Analysis (Straatemeier, et. al., 2008; Vaiopoulou, et. al., 2017). Findings from this research suggest that latent groups emerged from the analysis were not associated with the hypothesized mental models. This method, combined with catastrophe theory can provide an additional way to re-test the existence of the hypothesized stable entities. Theoretically, the assumed mental model, being stable, coherent and resistant to change, comprises the attractors of the system. Any shift from one to the other, would be a nonlinear transition and could be modeled as catastrophes. This analytic approach was applied to data taken from previous research employing cognitive variables in predicting or students' performance, specifically, formal reasoning and field dependence/independence had been found to explain students' mental representations on the structure of matter (Tsitsipis, et. al., 2012; Stamovlasis, et. al., 2013). LCA reveal three latent classes or potential attractors: One was the correct scientific model and two antecedent hypothetical models. Interestingly, transitions occurred only when the scientific model was used as one of the attractor regions, while this did not occur between the other antecedent hypothetical models. This means that the scientific model emerges from the interaction process (involving neo-Piagetian constructs) and it is not a product of a linear transformation. Contributing to this theoretical debate and based on the analysis of the particular data, nonlinear dynamics support the fragmented knowledge hypothesis. Of course, further exploration and research is needed to other data and subject matters; however, this present evidence challenges the theoretical premises of coherent mental models in learning processes, which the main stream research and practice has been based on for the last decades. Therefore, it opens new areas of investigations at the behavioral level towards understanding learning processes. It should be reminded that reforming theoretical premise is an endogenous and the core process in scientific inquiry according to Popper's epistemology (Popper, 1983), and under this view the added value of CDS entails new perspectives for theory development.

Summarizing, the catastrophe theory applied to empirical data taken from students' learning and conceptual understanding in sciences revealed nonlinearity in cognitive performance. This has important implications for theories of learning (and teaching). The new research methodologies in line with CDS view provide better interpretations of phenomena 
occurring within school processes and contribute to theory development. Lastly, it is important to point out here, that the above effort is a minimal piece of inquiry within a plethora of contributions with new ideas, methods and research findings fostering CDS meta-theory. The new stream is growing in educational research challenging the traditional epistemologies and aiming towards the Paradigm Shift.

\section{References}

Bunge, M. (1979). Causality and Modern Sciences. New York: Dover Publications, Inc.

Cleick, J. (2008). Chaos: Making a New Science. NY: Penguin.

Cobb L (1998). An Introduction to Cusp Surface Analysis." Technical report, Aetheling Consultants, Louisville, CO, USA. Available in: http://www.aetheling.com/models/cusp/Intro.htm.

diSessa A. A., (1988), "Knowledge in pieces", in G. Forman and P. B. Pufall (Eds.), Constructivism in the computer age (pp. 49-70), Hillsdale, NJ: Lawrence Erlbaum Associates, Inc.

Fleener, J. (2002). Curriculum dynamics: Recreating heart. New York: Peter Lang.

Gonida, E. \& Stamovlasis, D. (2016). Maternal involvement in homework, student goal orientations and achievement in Language and Mathematics: Supporting autonomy and avoiding interference. 15th International Conference on Motivation), August 24-27, Thessaloniki.

Grasman R. P., van der Maas H.L.J, Wagenmakers E.J. (2009). Fitting the Cusp Catastrophe in R: A cusp Package Primer. Journal of Statistical Software, 32, 1-27.

Guastello, S. J. (2002). Managing Emergent Phenomena. Nonlinear Dynamics in Work Organizations. Mahwah, New Jersey: Lawrence Erlbaum Associates, Publishers.

Guastello, S. J. (2011). Discontinuities and catastrophes with polynomial regression. In S. Guastello and R. Gregson (Eds.), Nonlinear Dynamics Systems Analysis for the Behavioral Sciences Using Real Data, (pp. 252-180). New York: CRC Press.

Johnson, P. M., \& Papageorgiou, G. (2010). Rethinking the introduction of particle ideas: A substance based framework. Journal of Research in Science Teaching, 47, 130-150.

Koopmans, M. (2014a). Nonlinear Change and the black box approach in educational research. Nonlinear Dynamics, Psychology and Life Science, 18, 5-22.

Koopmans, M. (2014b). Change, Self-organization and the Search for Causality in Educational Research and Practice. Complicity: An International Journal of Complexity and Education, 11, 20-39.

Lawson, A. E., \& Thompson, L. D. (1988). Formal reasoning ability and misconceptions concerning genetics and natural selection. Journal of Research in Science Teaching, 25, 733-746.

Maxwell, J. A. (2004). Causal explanation, qualitative research, and scientific inquiry in education. Educational Researcher, 33, 3-11.

Molenaar, P.C. \& Oppenheimer, L. (1985). Dynamic models of development and the mechanistic-organismic controversy. New Ideas in Psychology, 3, 233-242.

Nicolis, G. \& Nicolis, C. (2007). Foundations of Complex Systems. Singapore: World Scientific.

Papageorgiou, G. \& Johnson, P. (2005). Do particle ideas help or hinder pupils' understanding of phenomena? International Journal of Science Education, 27(11), 1299-1317.

Pascual-Leone, J. (1969). The encoding and decoding of symbols by children: A new experimental Paradigm and neo-Piagetian model. Journal of Experimental Child Psychology, 8, 328-355.

Pascual-Leone, J. (1970). A mathematical model for the transition rule in Piaget's developmental stages. Acta Psychologica, 32, 301-345.

Poston, T. \& Stewart, I. (1978). Catastrophe theory and its applications. New York: Dover Publications.

Stamovlasis, D. (2006). The Nonlinear Dynamical Hypothesis in Science Education Problem Solving: A Catastrophe Theory Approach. Nonlinear Dynamics, Psychology and Life Science, 10 (1), 37-70.

Stamovlasis, D. (2010). Methodological and epistemological issues on linear regression applied to psychometric variables in problem solving: Rethinking variance. Chemistry Education, Research and Practice, $11,59-68$.

Stamovlasis, D. (2011). Nonlinear dynamics and Neo-Piagetian Theories in Problem solving: Perspectives on a new Epistemology and Theory Development. Nonlinear Dynamics, Psychology and Life Science, 15, 145173. 
Stamovlasis, D. (2016). Catastrophe theory: Methodology, epistemology and applications in learning science. In M. Koopmans and D. Stamovlasis (Eds), Complex Dynamical Systems in Education: Concepts, Methods and Applications (pp. 141-175). Switzerland: Springer Academic Publishing.

Stamovlasis, D. \& Gonida, E. (2017). Dynamic effects of Performance-Avoidance Goal Orientation on Student Achievement in Language and Mathematics (working paper).

Stamovlasis, D. \& Koopmans, M. (2014). Editorial Introduction: Education is a Dynamical System. Nonlinear Dynamics, Psychology and Life Science, 18(1), 1-4. (Special Issue: Nonlinear Dynamics in Education).

Stamovlasis, D. \& Gonida, E. (2017). Dynamic effects of Performance-Avoidance Goal Orientation on Student Achievement in Language and Mathematics (working paper)

Straatemeier, M., van der Maas, H.L.J., \& Jansen, B.R.J. (2008). Children's knowledge of the earth: A new methodological and statistical approach. Journal of Experimental Child Psychology, 100, 276-296.

Thom, R. (1975). Structural stability and morphogenesis. New York: Benjamin-Addison-Wesley.

Tsaparlis, G. (2005). Non-algorithmic quantitative problem solving in university physical chemistry: A correlation study of the role of selective cognitive factors. Research in Science \& Technological Education. 23, 125-148.

Tsaparlis, G. \& Angelopoulos, V. (2000). A model of problem solving: Its operation, validity and usefulness in the case of organic-synthesis problems. Science Education, 84, 131-153.

Tsitsipis, G., Stamovlasis, D., \& Papageorgiou, G. (2010). The effect of three cognitive variables on students' understanding of the particulate nature and the changes of state of matter. International Journal of Science Education, 32, 8, 987-1016.

Tsitsipis, G., Stamovlasis, D. \& Papageorgiou, G. (2012). A probabilistic model for students' errors and misconceptions on the structure of matter in relation to three cognitive variables, International Journal of Science and Mathematics Education., 10, 777-802.

Vaiopoulou, J., Stamovlasis, D. \& Papageorgiou, G. (2017). New perspectives for theory development in science education: Rethinking mental models of force in primary school. In R.V. Nata (Ed.). Progress in Education, Volume 47 (pp.1-16). New York: Nova Science Publishers, Inc.

Vaiopoulou, J., Papageorgiou, G., \& Stamovlasis, D. (2017). Enhancing theoretical premises on the nature of students' mental representation of science concepts: The case of Force. In Proceedings of the International conference 'Quality and Equity in Education: Theories, Applications and Potentials' (pp. 123-128). Nicosia: Erasmus+, University of Cyprus.

van Geert, P. (2003). Dynamic systems approaches and modeling of growth developmental processes. In J. Valsiner \& K. J. Connolly (Eds.), Handbook of developmental psychology (pp. 640-672). London: Sage.

Van der Maas, H. L. J., \& Molennar, P. C. (1992). Stagewise Cognitive Development: An Application of Catastrophe Theory. Psychological Review, 3, 395-417.

Van der Maas, H. L. J., Kolstein, R. \& van der Pligt, J. (2003). Sudden transitions in Attitudes. Sociological Methods and Research, 23, 125-152.

Vosniadou S. \& Brewer W. F. (1992). Mental models of the earth, A study of conceptual change in childhood. Cognitive Psychology, 24, 535-585.

Zeeman, E. C. (1976). Catastrophe Theory. Scientific American, 4, 65-83.

\section{About the Author}

Dimitrios Stamovlasis, PhD, is Assistant Professor of Research Methodology and Applied Statistics for Social Science at the Aristotle University. His research interest they focus on methodological and epistemological issues of contemporary social sciences, nonlinear dynamics, complexity, catastrophe theory, entropy, and related fields. Correspondence: stadi@auth.gr.

(C) Copyright 2017. The author, DIMITRIOS STAMOVLASIS, assigns to the University of Alberta and other educational and non-profit institutions a non-exclusive license to use this document for personal use and in courses of instruction provided that the article is used in full and this copyright statement is reproduced. The authors also grant a non-exclusive license to the University of Alberta to publish this document in full on the World Wide Web, and for the document to be published on mirrors on the World Wide Web. Any other usage is prohibited without the express permission of the authors. 